\title{
Organ of Interest
}

National Cancer Institute

\section{Source}

National Cancer Institute. Organ of Interest. NCI Thesaurus. Code C150897.

The identification of an organ that may contain a tumor, lesion or site of disease, and

may be part of the objective assessment of the response to the therapeutic intervention. 